\title{
Challenges in application of hybrid switched control to digital hydraulic motors
}

\author{
I. Manganas ${ }^{1}$ T.O. Andersen ${ }^{2}$ P. Johansen ${ }^{2}$ L. Schmidt ${ }^{2}$ \\ ${ }^{1}$ E-mail: manganas.ioannis@gmail.com \\ ${ }^{2}$ Fluid Power and Mechatronic Systems, Department of Energy Technology, Aalborg University, Pontoppidanstraede \\ 111, 9220 Aalborg, Denmark. E-mail: $\{$ pjo, Isc, toa $\}$ @et.aau.dk
}

\begin{abstract}
In this paper, the challenges regarding the application of a switched control approach to a digital displacement machine $\left(\mathrm{DDM}^{\circledR}\right)$ are discussed. The system under consideration is initially presented. Subsequently, the reasons for considering the design and application of the specific, switched controller are discussed. Finally, the challenges are presented, which could function as future research.
\end{abstract}

Keywords: hydraulics, digital hydraulics, hydraulic motor, digital displacement, switched control

\section{Introduction}

Energy efficiency and reliability are the main drivers for the development of novel hydraulic actuators. To this end, applications where the actuation of fluid power components is based on on/off valves have emerged. One example is power take-off systems, used in wave energy converters, studied in ? and ?. Another example is the digital displacement machine $\left(\mathrm{DDM}^{\circledR}\right)$ from ?. In the following, when the term DDM is used, it refers to $\mathrm{DDM}^{\circledR}$. A similar approach, whose main difference lies in the technology of the valves, is by ?. It is an axial piston pump where instead of a swash plate, the displacement is controlled via independent pressurization or de-pressurization of each piston chamber. To this end, each cylinder is equipped with two onoff valves; one connecting the control volume in the chamber to high pressure and the other to low pressure. This technology has the potential for increased efficiency, since for low displacement values not every cylinder is exposed to a high pressure difference ?.

Control of this machine can become rather complicated and application dependent. One reason is the binary inputs to the on-off valves. Another reason arises from the mode of operation, namely full or partial stroke. The former leads to increased efficiency, however for low displacement the output can become discontinuous and unacceptable time delay is introduced ?. It has been shown in ? that for high operation speed, the dynamics of the DDM can be neglected, and a duty ratio approximation yields controllers resulting in very satisfactory results, designed using well known techniques.

In the case the speed and displacement are high for the majority of the operation region, full stroke operation is preferable. In ? the discrete time dynamics of the DDM were taken into consideration alongside those of the plant, and LQR controllers were designed. Since the sampling time is dependent on the speed of the shaft, the system is brought in the position, or shaft angle, domain. Operation at zero speed cannot occur this way. This fact renders applications requiring change of rotation direction as well as operation at standstill infeasible.

For low speed-high torque applications, a different strategy is needed. Furthermore, reversal of direction and position set point regulation require the shaft speed being zero, even during a very small time interval. A potential application scenario is a hydraulic winch on an offshore platform ?,?,?. Since the number 
of cylinders is predetermined and the control inputs are binary, it is not difficult to think of different combinations as different discrete states with distinct equilibrium points. As a result a hybrid type of controller could be an alternative to a discrete, maybe predictive, controller.

In the field of switched electronics, especially when the number of the different switching states is low, controllers designed using a hybrid framework have been designed. The majority of controllers in industry are designed upon a duty ratio approximation as (S)PWM controllers. However, the design of hybrid controllers approaches the problem in a way that can be used for the topic of this article. Different hybrid and optimal control schemes are compared in ? and ? for the buck and boost converter respectively. In these works, the hybrid methodology refers to taking into consideration the discontinuous switching, designing controllers based on the complete dynamics of the converter instead of a low frequency approximation. In ? and ?, the DC-DC boost converter is considered. The hybrid framework as presented in ? is used. The hybrid controller is based on the current switching state, as well as measurements of the continuous states in order to calculate the next switching state. In ?, the same hybrid control design framework is adapted to a single phase $\mathrm{DC} / \mathrm{AC}$ converter. The resulting controller is found in simulations to produce lower harmonic distortion than the PWM controller. The switched systems approach to designing hybrid controllers for DC-DC converters is examined in ?. The cases of the buck, boost and buck-boost converters are illustrated as examples. All the aforementioned works are based on systems with a low number of switching states, or modes, which is the consequence of the low number of switching elements. Owing to its intuitive procedure, the last approach is selected to be followed in the present article.

In this paper, the challenges for designing a hybrid switched controller for a DDM operating as a motor, are discussed. In Section 2, the DDM operating as motor is described and the nonlinear differential equations that describe its components are presented. In Section 3 , the attempted control approach is presented. In Section 4 , the challenges that hinder the direct applicability of the selected control approach to the given system are discussed.

\section{Description of the system}

\subsection{Description and operation principle}

The objective is either shaft angle regulation or speed control. However, both directions of rotation might be required. In order to keep the complexity of the design low, the number of cylinders is $N=3$. A DDM like this is illustrated in Figure 1.

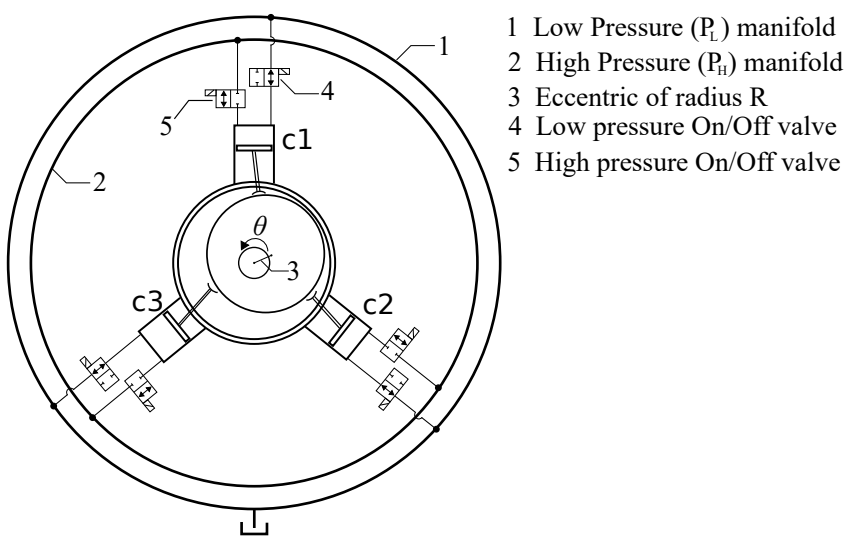

Figure 1: A DDM consisting of 3 cylinders.

The principle of operation for the so-called creep mode, as described in ?, is the following:

- Decide of a direction of rotation.

- Depending on the direction, a cylinder that contributes positive torque towards that direction, is pressurized.

- Block all ports of all cylinders.

- When the system is in equilibrium, a cylinder that contributes negative torque is depressurized.

- At the next equilibrium point, the procedure is repeated.

Modifications to this algorithm can be simultaneous pressurization or de-pressurization of multiple cylinders and not waiting to reach an equilibrium point for the next step in the algorithm. In this document, the case where only one cylinder can be active at a time is investigated. Denoting for each cylinder the cases:

- 1: High pressure valve is open. The control volume of the cylinder is pressurized.

- -1: Low pressure valve is open. The control volume of the cylinder is de-pressurized.

- 0: Both valves are closed. No flow comes in or goes out of the chamber of the cylinder.

The allowed combinations, which constitute the discrete states, for the three cylinders are shown in Table 1.

It is of interest to design a switching signal that commands the change of states $S_{j}, j=0,1,2, \ldots, 6$, so that the resulting equilibrium point is as close as possible to the reference. To this end, the equilibrium points of 
Table 1: Table of allowed states for the three cylinders

\begin{tabular}{c|ccc} 
State & C3 & C2 & C1 \\
\hline$S_{0}$ & 0 & 0 & 0 \\
$S_{1}$ & 0 & 0 & 1 \\
$S_{2}$ & 0 & 1 & 0 \\
$S_{3}$ & 1 & 0 & 0 \\
$S_{4}$ & 0 & 0 & -1 \\
$S_{5}$ & 0 & -1 & 0 \\
$S_{6}$ & -1 & 0 & 0
\end{tabular}

the system in these states must be identified, preferably in closed form. If more combinations were allowed, it is expected that more equilibrium points will be available, increasing the output resolution of the motor. The possible combinations are $m^{N}-2$, where $m=3$, the number of possible values for the state of each valve of a cylinder $0,-1$ or 1 . The 2 combinations that are discarded are 111 and $-1-1-1$ since the former does not produce torque and the latter corresponds to the idle state. It is noted here that the idle state is an important feature of the DDM technology for increased efficiency, but for the scope of this article where the DDM operates as a motor, it is not used.

\subsection{Model of the system}

Modelling of the system follows the work in ?. The nonlinear model of the system is consisted of:

- Piston displacement:

$$
\begin{aligned}
x_{p} & =R\left[1-\cos \left(\theta_{i}\right)\right] \\
\theta_{i} & =\theta+\frac{2 \pi}{N}(i-1) i=1,2, . ., N=3
\end{aligned}
$$

where $R$ is the eccentric radius and $\theta$ is the shaft angle.

- Torque contribution of each cylinder:

$$
\begin{aligned}
\tau_{i} & =P_{i} D_{i} \\
D_{i} & =V_{d} \sin \left(\theta_{i}\right)
\end{aligned}
$$

where $P_{i}$ is the pressure of the control volume inside cylinder's $i$ chamber and $V_{d}=A * R$ with $A$ the piston area. The total torque contribution is:

$$
\begin{aligned}
\tau & =\sum_{i=1}^{N} \tau_{i}= \\
& =V_{d}\left[P_{1} \sin \left(\theta_{1}\right)+P_{2} \sin \left(\theta_{2}\right)+P_{3} \sin \left(\theta_{3}\right)\right]
\end{aligned}
$$

- Chamber pressure:

$$
\dot{P}_{i}=\frac{\beta_{e f f}}{V_{0}+V_{d}\left[1-\cos \left(\theta_{i}\right)\right]}\left[-V_{d} \dot{\theta} \sin \left(\theta_{i}\right)+q_{H}-q_{L}\right]
$$

The temperature and pressure dependent effective oil bulk modulus is considered to be constant. However, this is not very realistic, since the chamber pressure becomes low. The terms $q_{H}$ and $q_{L}$ correspond to the flow through the high and low pressure valves' orifices respectively and when they are active:

$$
\begin{aligned}
q_{H} & =K_{v} \sqrt{P_{H}-P_{i}} \\
q_{L} & =K_{v} \sqrt{P_{i}-P_{L}}
\end{aligned}
$$

As stated in ?, there exists a system that protects against cavitation and furthermore, the valves can open passively. This fact adds to the complexity and difficulty finding equilibrium points.

In order to keep the order of the system low, a rough approximation has been made regarding the dynamics of the on-off valves. It has been assumed that they are infinitely fast, ie. $\frac{x_{v}(t)}{x_{v, \max }} \approx \frac{u_{v}(t)}{u_{v, \max }}$ for a wide range of input voltage frequencies. $x_{v}(t)$ refers to the instantaneous position of the on-off valve, while $u_{v}(t)$ refers to its input voltage. This is not necessarily true, since the settling times range in the order of ms, however compared to the time constant of the shaft, they are neglected.

- The shaft dynamics, using Newton's second law and Equation (4), can be written as:

$$
\begin{aligned}
\ddot{\theta} & =-\frac{b}{J} \dot{\theta}-\frac{\tau_{\text {load }}}{J}+ \\
& +\frac{V_{d}}{J}\left[P_{1} \sin \left(\theta_{1}\right)+P_{2} \sin \left(\theta_{2}\right)+P_{3} \sin \left(\theta_{3}\right)\right]
\end{aligned}
$$

It is assumed that $\tau_{\text {load }}$ is known and always positive for this application scenario.

- For the state vector $\mathbf{x}=\left[\begin{array}{lllll}x_{1} & x_{2} & P_{1} & P_{2} & P_{3}\end{array}\right]^{T}$, the dynamic equations describing the system are shown in Equations (9)-(11) (see top of page 4).

\subsubsection{Equilibrium when all valves are closed}

An initial investigation suggests finding the equilibrium points of the system under constant, known load. This amounts to finding tuples $\left(x_{1}, P_{1}, P_{2}, P_{3}\right)$ satisfying:

$$
\begin{aligned}
P_{1} \sin \left(x_{1}\right)+ & P_{2} \sin \left(x_{1}+\frac{2 \pi}{3}\right)+ \\
& +P_{3} \sin \left(x_{1}+2 \frac{2 \pi}{3}\right)=\frac{\tau_{l o a d}}{V_{d}}
\end{aligned}
$$

where $P_{L} \leq P_{i} \leq P_{H}$. There can be a case where $P_{i}>P_{H}$ for the valves, see $?$, but this situation is not considered because if the high pressure valve opens, 


$$
\begin{aligned}
& \dot{x}_{1}=x_{2} \\
& \dot{x}_{2}=-\frac{b}{J} x_{2}+\frac{V_{d}}{J}\left[P_{1} \sin \left(x_{1}\right)+P_{2} \sin \left(x_{1}+\frac{2 \pi}{3}\right)+P_{3} \sin \left(x_{1}+2 \frac{2 \pi}{3}\right)\right]-\frac{\tau_{\text {load }}}{J} \\
& \dot{P}_{i}=\frac{\beta_{\text {eff }}}{V_{0}+V_{d}\left[1-\cos \left(x_{1}+(i-1) \frac{2 \pi}{3}\right)\right]}\left[-x_{2} V_{d} \sin \left(x_{1}+(i-1) \frac{2 \pi}{3}\right)+q_{H, i}-q_{L, i}\right], \quad i=1,2,3
\end{aligned}
$$

the torque contribution will be decreased since $P_{i}$ will eventually become equal to $P_{H}$.

Finding the chamber pressures and shaft angle as a function of the load torque can be a difficult task. One way to find a closed form solution can be the following procedure:

- Assume that the load is not attached, the high pressure valves are closed and the low pressure valves are open for all cylinders.

- At an initial shaft angle $x_{1,0}$, all valves are closed, without attaching the load.

- With the shaft angle equal to $x_{1,0}$, the load is attached.

For each chamber, the pressure dynamics are described by the flow continuity equation:

$$
\dot{P}_{i}=-\frac{\beta_{e f f}}{V_{i}} \dot{V}_{i}
$$

Under the assumption that $\beta_{\text {eff }}$ is constant, using the separation of variables to solve the differential Equation (13) for each cylinder:

$$
P_{f, i}=P_{0, i}+\beta_{e f f} \ln \left(\frac{V_{0, i}}{V_{f, i}}\right)
$$

where $P_{f, i}$ and $P_{0, i}$ stand for the final and initial value of the chamber pressure of cylinder $i=1,2,3$, respectively. The same is valid for the volumes $V_{f, i}$ and $V_{0, i}$. Substituting, using the assumptions above:

$$
\begin{aligned}
P_{0, i} & =P_{L} \\
V_{0, i} & =V_{0}+V_{d}\left[1-\cos \left(x_{1,0}+\frac{2 \pi}{N}(i-1)\right)\right] \\
V_{f, i} & =V_{0}+V_{d}\left[1-\cos \left(x_{1, f}+\frac{2 \pi}{N}(i-1)\right)\right]
\end{aligned}
$$

Substituting $P_{i}$ in Equation (12) with Equation (14) for each cylinder, a condition that needs to be satisfied for $x_{1, f}, x_{1,0}$ and $\tau_{\text {load }}$ is produced. The value of $x_{1, f}$ at equilibrium is required to be found. It is not easy to find a closed form for the equilibrium shaft angle, given an initial value and a load torque. As a result, this is implemented numerically. A loop where angle values in the interval $[0,2 \pi]$, as well as chamber pressures for each cylinder $P_{i} \in\left[P_{L}, P_{H}\right]$ is written in Matlab. All possible values for the angle are tested to check whether they satisfy the condition given by Equation (12), with Equation (14) substituted. This procedure is in other words solving both sides of Equation (12) for all angles and finding the points where they are equal, for a given load and initial conditions.

For the case where 3 different initial shaft angles are considered, the following plots of Equation (12) are shown in Figures 2, 4 and 5. The displacement volume for the simulation was selected as $V_{d}=6.3662 \cdot 10^{-4}$ $\left[\frac{\mathrm{m}^{3}}{\mathrm{rev}}\right]$ and the load torque $\tau_{\text {load }}=8.75 \cdot 10^{3}[\mathrm{~N} \cdot \mathrm{m}]$. In the figures, the shaft angle is iterated between $0-2 \pi$ [rad] and the equilibrium points lie on the intersection of the total actuation torque line (blue colour) and the load torque line (red colour).

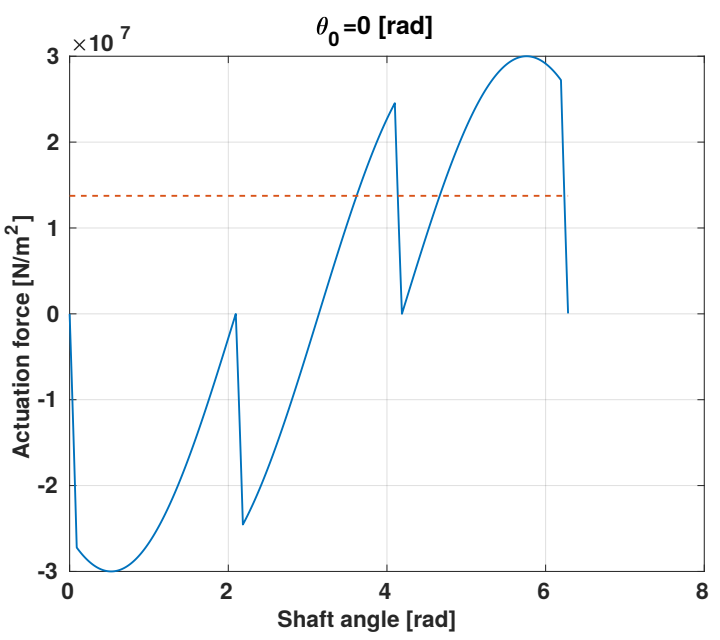

Figure 2: Equilibrium points when initial angle is 0 [rad].

It can be seen that depending on the initial value of the shaft angle, different, and in some case, multiple equilibrium points are present. Despite this fact, if the followed approach is correct, the equilibrium points could be investigated in regard to their stability property using Lyapunov's indirect method. However, simulation results on the nonlinear system using the same 


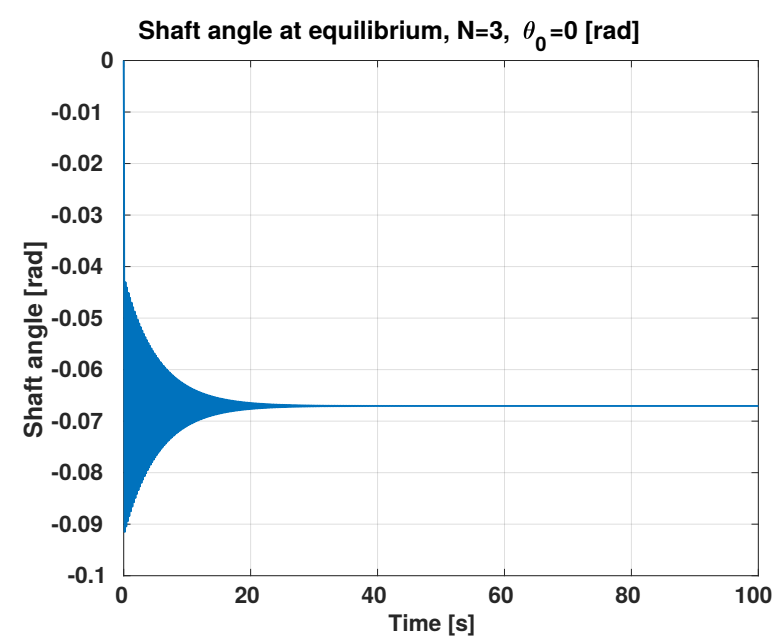

Figure 3: Equilibrium point when initial angle is 0 $[\mathrm{rad}]$, from the simulation model.

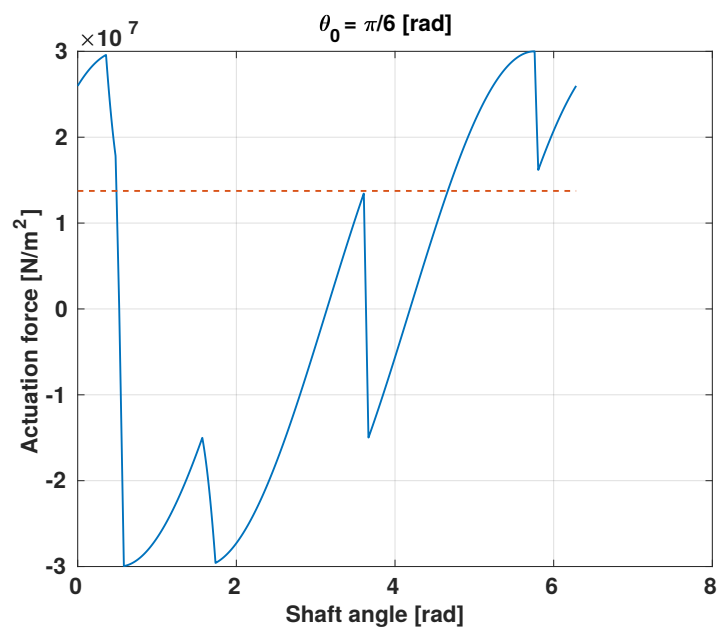

Figure 4: Equilibrium points when initial angle is $\frac{\pi}{6}$ $[\mathrm{rad}]$.

parameters do not agree in the value of the equilibrium point for the shaft angle. For example, in Figure 3 a simulation is used to calculate the equilibrium point when the initial angle equals to 0 [rad]. The same parameters have been used for the DDM, when the equilibrium points are analytically calculated, as described. This result is to be compared with Figure 2, see also discussion in section 4.1. The parameters used are shown in Table 2.

\section{Switched control}

A system that switches between dynamic behaviors in different parts of the state space is called a switched system. Switched systems are a class of hybrid systems

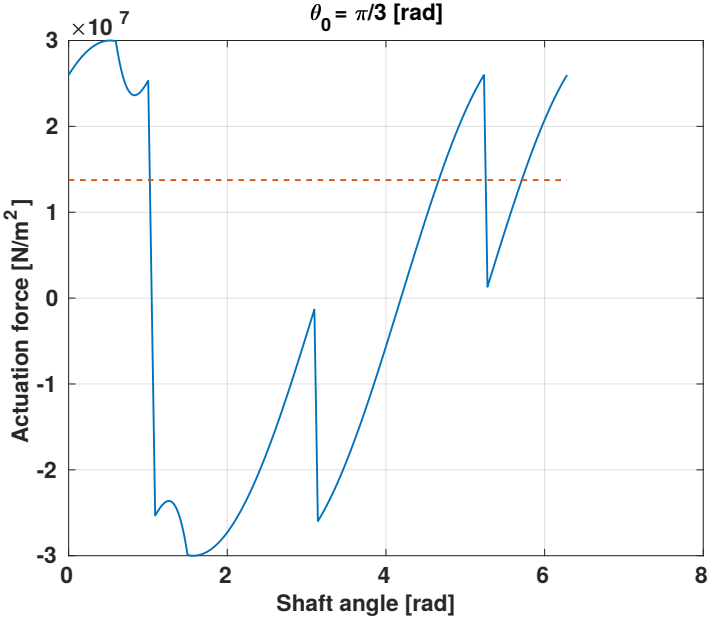

Figure 5: Equilibrium points when initial angle is $\frac{\pi}{3}$ [rad].

Table 2: Parameters used in simulations.

\begin{tabular}{c|cc} 
Parameter & Value & Unit \\
\hline$V_{0}$ & 0.250 & $l$ \\
$V_{d}$ & 0.63662 & $l$ \\
$R$ & 25 & $\mathrm{~mm}$ \\
$P_{H}$ & 310 & $\mathrm{bar}$ \\
$P_{L}$ & 10 & $\mathrm{bar}$ \\
$b$ & 200 & $\mathrm{Nm} \mathrm{s} / \mathrm{rad}$ \\
$J$ & 534 & $\mathrm{~kg} \mathrm{~m} m^{2}$ \\
$K_{v}$ & $10^{-6}$ & $\mathrm{~m}^{2} \mathrm{~Pa}^{-1} s^{-1}$ \\
$N$ & 3 & \\
$\beta_{\text {eff }}$ & 7000 & $\mathrm{bar}$ \\
$\tau_{\text {load }}$ & 8.75 & $\mathrm{kNm}$ \\
& &
\end{tabular}

where no jumps in the continuous states occur when switching between discrete states. A family of systems, suitable for modeling the DDM operating as a motor linearized about a point of operation, are of the form:

$$
\dot{\mathbf{x}}=\mathbf{A}_{i} \mathbf{x}+\mathbf{b}_{i}
$$

It is important to know whether switching can result in an unstable system and furthermore, which combination of the subsystems leads to the desired performance.

The fact that the valves in practice have dynamics would impose a minimum dwell time criterion, so that the valve spool position has finished its transition from fully open to fully closed, or vice versa. This means that a switching between discrete states cannot occur until the system has stayed in the current discrete state for time at least equal to the dwell time. However, the dynamics of the valves are considered significantly faster and are neglected. 
Stability of switched systems can be proven by a common Lyapunov function ?, Theorem 2.1, with time derivative negative definite along the trajectories of all subsystems, independently of the switching signal. It is difficult to satisfy all the conditions for the common Lyapunov function, especially in the case that the number of subsystems is 7 .

However, if there can be a Lyapunov function for a convex combination of the subsystems, then the switching system is uniformly stable ?, Corollary 2.3. Using this result, it will be assumed that a convex combination exists and a Lyapunov function will be used to investigate the conditions under which the switched system is uniformly asymptotically stable. This will help with the design of the switching signal.

Since the system in question is nonlinear, selecting a Lyapunov function candidate can be a non trivial task. However, for linear systems, there exists a way to design Lyapunov functions, which can be a good start for the investigation. As a result, the system is linearized about points of operation, which should correspond to the equilibrium points at each discrete state $S_{j}, j=0,1, \ldots, 6$. As a result, the system is treated as if it was linear and the controller is designed based on this model. Hopefully, all initial conditions lie in the vicinity of the region of attraction of the equilibrium point of the plant-controller system.

It is known that for linear systems of the form:

$$
\dot{\mathbf{x}}=\mathbf{A x}
$$

where A is strictly Hurwitz, a suitable selection for a Lyapunov function is:

$$
V(\mathbf{x})=\mathbf{x}^{T} \mathbf{P} \mathbf{x}
$$

where $\mathbf{P}$ is a symmetrical and positive definite matrix. The derivative of $V(\mathbf{x})$ along the trajectories of the system with dynamics described in Equation (16) becomes:

$$
\dot{V}(\mathbf{x})=<\nabla V(\mathbf{x}), \mathbf{x}>=\mathbf{x}^{T}\left(\mathbf{A}^{T} \mathbf{P}+\mathbf{P A}\right) \leq-\mathbf{Q}
$$

where $\mathbf{Q}$ is a positive definite matrix, usually selected to be the identity matrix $\mathbf{I}$.

The family of systems in this case does not need to have strictly Hurwitz matrices, however, there should be a convex combination that achieves this property. Assuming that this combination exists, denoted as $\mathbf{A}_{\lambda}$, a Lyapunov function candidate is, according to Lemma $1, ?$ :

$$
\begin{aligned}
V(\mathbf{x}) & =\mathbf{x}^{T} \mathbf{P} \mathbf{x} \\
\dot{V}(\mathbf{x}) & =\mathbf{x}^{T}\left(\mathbf{A}_{\lambda}^{T} \mathbf{P}+\mathbf{P} \mathbf{A}_{\lambda}\right) \mathbf{x}= \\
& =\min _{i \in K} \mathbf{x}^{T}\left(\mathbf{A}_{i}^{T} \mathbf{P}+\mathbf{P} \mathbf{A}_{i}\right) \mathbf{x} \\
& \leq \min _{\lambda \in \Lambda} \mathbf{x}^{T}\left(\mathbf{A}_{\lambda}^{T} \mathbf{P}+\mathbf{P} \mathbf{A}_{\lambda}\right) \mathbf{x}<0
\end{aligned}
$$

where $K$ is the set $\{0,1,2, \ldots, 6\}$. An element in $K$ denotes the active subsystem. $\mathbf{A}_{\lambda}$ denotes the convex combination of the subsystem dynamics matrices $\mathbf{A}$ :

$$
\mathbf{A}_{\lambda}=\sum_{i=0}^{6} \lambda_{i} \mathbf{A}_{i}, \quad \lambda_{i} \leq 1, \quad \sum_{i=0}^{7} \lambda_{i}=1
$$

For the case where there exists $\mathbf{A}_{\lambda}$ Hurwitz, then according to ?, Lemma 1, a switching signal that stabilizes the origin as equilibrium point can be designed as:

$$
\sigma(\mathbf{x})=\arg \min _{i \in K} \mathbf{x}^{T} \mathbf{P A} \mathbf{x}
$$

\subsection{The system under consideration}

For the family of systems described by Equation (15), an appropriate Lyapunov function can be:

$$
V(\mathbf{e})=\mathbf{e}^{T} \mathbf{P e}
$$

where $\mathbf{e}=\mathbf{x}-\mathbf{x}_{\mathbf{e}}$ with $\mathbf{x}_{\mathbf{e}}$ an attainable equilibrium point. What is left is to find assumptions that will render the selected equilibrium point Uniformly Asymptotically Stable, preferably Globally ((G)UAS). To this end, a switching logic and some assumptions have to be made. The derivative of the Lyapunov function will help with both these requirements. Along the trajectories of the system in Equation (15):

$$
\begin{aligned}
\dot{V}(\mathbf{e}) & =2 \mathbf{e}^{T} \mathbf{P} \dot{\mathbf{}} \\
& =2 \mathbf{e}^{T} \mathbf{P}\left(\mathbf{A}_{\sigma} \mathbf{x}+\mathbf{b}_{\sigma}\right) \\
& =2 \mathbf{e}^{T} \mathbf{P}\left(\mathbf{A}_{\sigma} \mathbf{x}+\mathbf{b}_{\sigma}\right)+\mathbf{e}^{T} Q_{\sigma} \mathbf{e}-\mathbf{e}^{T} Q_{\sigma} \mathbf{e} \\
& =\min _{i \in K} \mathbf{e}^{T}\left[2 \mathbf{P}\left(\mathbf{A}_{\mathbf{i}} \mathbf{x}+\mathbf{b}_{i}\right)+Q_{i} \mathbf{e}\right]-\mathbf{e}^{T} Q_{\sigma} \mathbf{e} \\
& =\min _{\lambda \in \Lambda} \mathbf{e}^{T}\left[2 \mathbf{P}\left(\mathbf{A}_{\lambda} \mathbf{x}+\mathbf{b}_{\lambda}\right)+Q_{\lambda} \mathbf{e}\right]-\mathbf{e}^{T} Q_{\sigma} \mathbf{e} \\
& =\min _{\lambda \in \Lambda} \mathbf{e}^{T}[2 \mathbf{P} \mathbf{A}_{\lambda} \mathbf{e}+\underbrace{2 \mathbf{P} \mathbf{A}_{\lambda} \mathbf{x}_{e}+2 \mathbf{P b}_{\lambda}}_{0}+Q_{\lambda} \mathbf{e}]- \\
& -\mathbf{e}^{T} Q_{\sigma} \mathbf{e} \\
& \leq-\mathbf{e}^{T} \mathbf{Q}_{\sigma} \mathbf{e}
\end{aligned}
$$

In order to get to the negative definite conclusion for $\dot{V}(\mathbf{e})$ :

- The second equality comes from the fact that $x_{e}$ is a static equilibrium point, belonging in the set of attainable equilibrium points.

- Assume that there exists $\mathbf{Q}_{\sigma}>0$.

- Assume that there exists a convex combination $\mathbf{A}_{\lambda}$ which is Hurwitz. 
- Assume that there exists $\mathbf{P}: \mathbf{P}^{T}=\mathbf{P}>0$. The last three assumptions take the last equality to the inequality, and the conclusion that $\mathbf{x}_{e}$ is $(\mathrm{G}) \mathrm{UAS}$. The global characterization cannot be guaranteed, since the system is nonlinear in nature and this is a linearization about these equilibrium points. There can be other equilibrium points outside the small neighborhood where the linearization is valid.

- In the last equality, the 0 result comes from the fact that $\mathbf{x}_{e}$ satisfies the condition of being an equilibrium point of the linearized system, and as a result, $\dot{x}_{e}=0$.

The switching signal can be selected as:

$$
\sigma\left(\mathbf{x}, \mathbf{x}_{e}\right)=\min _{i \in K} \mathbf{e}^{T}\left[2 \mathbf{P}\left(\mathbf{A}_{\mathbf{i}} \mathbf{x}+\mathbf{b}_{i}\right)+Q_{i} \mathbf{e}\right]
$$

since only one system $i$ can be active for a given time period and region of the state space. The selection is based on the Lyapunov function derivative, using the fourth equality.

Using this design, one finds the system dynamics matrices, $\mathbf{A}_{i}$, a convex combination of them $\mathbf{A}_{\lambda}$ which is Hurwitz, the attainable set of equilibrium points, then the matrices $\mathbf{Q}$ and $\mathbf{P}$. In ?, $\mathbf{Q}$ is found by utilizing the output matrix, since output feedback was used.

A somewhat different approach is made in ?. In that approach, the switching frequency can be lowered using a positive parameter, smaller than 1. However, every dynamics matrix $\mathbf{A}_{i}$ must be strictly Hurwitz and an LMI problem needs to be solved to find the matrix $\mathbf{P}$.

\section{Challenges}

Trying to apply the approach in Section 3 to the given system is straightforward. The very nature of the system poses difficulties. The system in question, described by Equations (9)-(11) is nonlinear. In order to apply the readily available results presented in Section 3 , the system is linearized. The points of linearization should be the equilibrium points at each different discrete state $i$. These states correspond to different positions for the valves of each cylinder, as shown in Table 1. An initial approach is to find the equilibrium points for these states. The following step is to find the eigenvalues of the dynamics matrices, $\mathbf{A}_{i}$ and find a convex combination that is Hurwitz.

\subsection{Equilibrium points}

As noted in Section 2.2.1, it is already difficult to find the equilibrium points when all valves are closed, or the state $S_{0}=000$, corresponding to a dynamics matrix $\mathbf{A}_{i}$. Obtaining the equilibrium points for the different states in closed form is difficult, more so when a chamber is pressurized, since the movement of the shaft affects the pressure in the chambers of the other cylinders. Simulation could be a solution, however, the effective bulk modulus should also be modeled as a nonlinearity. This is not effective for application, since a very high number of simulations should be realized, to cover the majority of initial conditions and loads. Another important prerequisite is that the equilibrium point should be stable. This can be checked using Lyapunov's indirect method.

\subsubsection{Linearization}

Assuming one has managed to find the equilibrium points in closed form, denoted as $\mathbf{x}_{e, i}, i=0,1, \ldots, 6$, the next step would be to linearize the nonlinear system to bring it to the form in Equation (16).

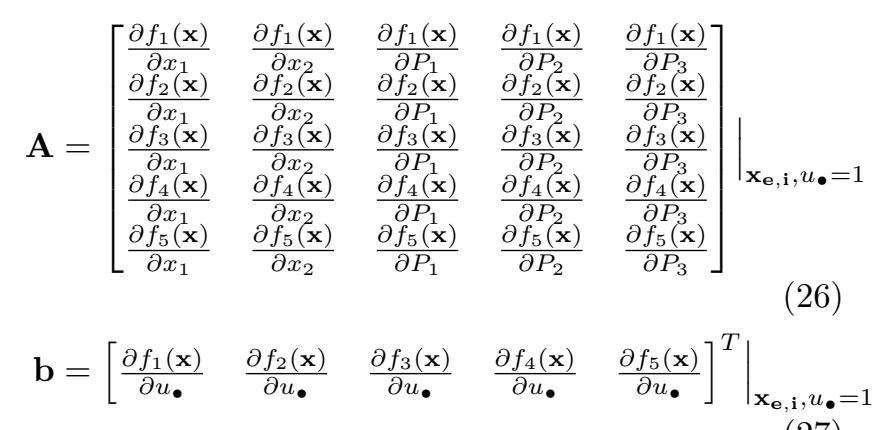

where $\bullet$ denotes which valve is open, the high or low line pressure.

The $\mathbf{A}$ matrices differ between discrete states when the valves are not open for a cylinder, since then, in the flow continuity equation, $q_{H}=q_{L}=0$. The elements of the dynamics matrices $\mathbf{A}_{i}$ are rather complicated due to the volume in the denominator in the flow continuity equation. This fact is however an advantage of the hybrid modeling and control, despite its complexity. If a linear controller with constraints was to be used, designed based on one linearized model, the flow through the orifice of each on-off valve for a cylinder would be linearized about a point. When the valve is off, there is no flow and as a result, the pressure dependence term in the dynamics matrix should be absent. In the case there was damping due to leakage, only this damping would be affected. However, when there is no damping, when the flow is equal to 0 , the pressure dynamics system is not dependent on its previous values. 


\subsection{Convex combination}

According to the assumptions for $(\mathrm{G}) \mathrm{UAS}$ of Section 3 , a convex combination needs to be found for the dynamics matrices of the linearized system. For this case, where only one valve can be on at a time, there exist 7 discrete states. This means that one should try to find $\lambda_{i}, i=0, . ., 6$ so that:

$$
\mathbf{A}_{\lambda}=\sum_{i=0}^{6} \lambda_{i} \mathbf{A}_{i}
$$

has eigenvalues with real part negative. However, if all matrices $\mathbf{A}_{i}$ are Hurwitz, this should not pose an obstacle.

\subsection{Passive opening of the valves}

The passive opening of the valves must be taken into consideration, since the equilibrium points will differ.

\section{Preliminary conclusions}

The main reasons why it is deemed difficult to apply a switching signal based on an optimization criterion for a DDM in motor mode, for creep operation are:

- The large number of discrete states, even for low number of cylinders. This makes it difficult to find a convex combination of the dynamics matrices.

- The difficulty in obtaining the set of attainable equilibrium points.

Different approaches are not ruled out, especially for speed control. A model predictive control (MPC) approach that takes into consideration the different combinations of the cylinders' states is presented in ?. However, the switched control method is based on proving Lyapunov stability, albeit on the linearized system, when specific assumptions hold.
Another issue might be the scope of the problem. For applications requiring Low Speed High Torque, or during creep mode operation, the requirement might not be strictly to have regulation around an equilibrium point. Alternatively, it might be to have only velocity control with reference provided by a human operator. The operator can act as the position regulator, closing the position loop. Thus, if only speed control is the requirement, around standstill, different techniques could be used involving lower complexity. The fact that the torque contribution of each cylinder is a function of the shaft angle, which can be measured, can be utilized for algorithms that are not dependent on state combinations and crisp data, but rather on the relationship between measured signals and possible outputs. Proving closed loop stability can be however as challenging.

Future research on hybrid control system design for DDM could focus on approximations that decrease the complexity stemming from the number of cylinders. Since operation at standstill and low speeds is essential for 4 quadrant operation, stability based closed loop control design remains relevant. This mode of operation cannot be captured by the closed loop techniques designed in ?, since either the sampling time $T_{s}=\frac{2 \pi}{N \omega}$ or the angle domain transformation $\frac{\partial f}{\partial \theta}=\frac{1}{\omega} \frac{d f}{d t}$ fails since $\omega=0$ at standstill or at direction reversal. On the other hand, if a stability based controller is designed, the hybrid framework can be used to prove stability for all operating conditions.

\section{Acknowledgments}

The research was supported by the research council of Norway, SFI Offshore Mechatronics, project number $237896 / 030$. 